\title{
Dietary patterns throughout childhood and associations with nutrient intakes
}

\author{
Victoria Cribb, Pauline Emmett and Kate Northstone* \\ School of Social and Community Medicine, University of Bristol, Oakfield Grove, Bristol BS8 2BN, UK
}

Submitted 22 February 2012: Final revision received 14 June 2012: Accepted 26 June 2012: First published online 14 September 2012

\begin{abstract}
Objective: To examine how the dietary patterns of children at various time points throughout childhood relate to estimated nutrient intakes.

Design: FFQ at 3, 4, 7 and 9 years of age were completed by mothers. Dietary patterns were identified cross-sectionally using principal component analysis; 'processed', 'health conscious' and 'traditional' patterns were consistently obtained. Correlations between pattern scores and nutrient intakes and proportions of variance in nutrients explained by the patterns were calculated.

Setting: Avon Longitudinal Study of Parents and Children (ALSPAC), Bristol, UK.

Subjects: Children provided data between 3 and 9 years of age ( $n 8010$ to 10023 ).

Results: Dietary patterns explained substantial proportions of the variance of the absolute intake for most nutrients $(>25 \%$ at 3 years of age, $>40 \%$ other ages). After energy adjustment, protein, fibre, $\mathrm{K}, \mathrm{Mg}, \mathrm{Fe}, \mathrm{Zn}$, folate, thiamin and vitamin $\mathrm{B}_{6}$ continued to be well explained. Strong correlations were observed between the 'processed' pattern and macronutrients including energy $(r=0 \cdot 481-0 \cdot 619)$, total fat $(r=0.529-0.662)$ and total sugar $(r=0 \cdot 475-0 \cdot 693)$. However correlations with most micronutrients were reversed after energy adjustment, suggesting that the 'processed' pattern is energy-dense but nutrient-poor. The 'health conscious' and 'traditional' patterns were strongly positively correlated with protein, fibre and most micronutrients, whether energy adjusted or not. Higher scores on these patterns were associated with a better nutrient profile.

Conclusions: Dietary patterns explain a reasonable amount of the variation in the nutrient content of diets. Higher scores on the 'health conscious' and 'traditional' dietary patterns were related to better nutrient profiles; conversely, with higher scores on the 'processed' pattern the nutrient profile was poorer.
\end{abstract}

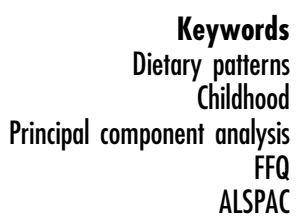

Diet has been associated with many health conditions such as CHD, obesity, diabetes and some types of cancer ${ }^{(1-5)}$. Substantial evidence also indicates that diet is important throughout childhood and adolescence, as it has been shown that dietary habits established during childhood are likely to track into adulthood ${ }^{(6)}$. It is therefore important to examine diet several times during childhood.

Various epidemiological studies that have examined diet and health relationships have concentrated primarily on either individual nutrients and/or foods ${ }^{(7)}$. These traditional methods have provided valuable evidence but have several methodological limitations; the high inter-correlations that exist between nutrients mean that the effects of a single nutrient may be hard to detect ${ }^{(8)}$ or distinguish from other nutrients. More recently, the focus has shifted towards exploring the whole diet to assess these relationships using dietary patterns. Individuals do not eat nutrients in isolation; they eat a variety of foods, consisting of a combination of nutrients, and the interaction of these nutrients can make it difficult to explore the individual effect of each nutrient ${ }^{(9)}$.
Therefore, it is not just the absence or presence of a particular food, but the variety and combination of foods in the diet that are important for optimal health ${ }^{(10))}$. Principal component analysis (PCA) is the most commonly used method to describe overall dietary patterns, using the correlations that exist between the foods eaten in combination. Such a method gives a summary of the foods/drinks consumed and therefore has the potential to provide a better indicator of disease risk ${ }^{(8)}$. It aims to better understand the complexities of diet and examine the associations between nutrition and health, in contrast to the traditional nutrient-based method ${ }^{(11)}$. Using PCA a score is obtained for each pattern which can be used to explore the relationships between the dietary patterns and either nutrient intakes or health outcomes ${ }^{(7,8)}$. Using dietary patterns in causal analyses has the potential to be more informative than focusing on individual nutrients or foods, and should inform the development of public health initiatives ${ }^{(12)}$ and be easier to translate into messages relevant to the dayto-day lives of individuals ${ }^{(13)}$. 
Research examining the associations between dietary patterns and nutrient intakes is limited. Previous work within the Avon Longitudinal Study of Parents and Children (ALSPAC) has examined such relationships in pregnant women and shown that scores on the 'health conscious' and 'traditional' patterns were positively related to all nutrients, while the 'processed' pattern was negatively associated with most nutrients except for energy, fats and sugar, all of which increased with higher scores $^{(14)}$. These relationships have not previously been investigated in children. Thus, the aim of the present study was to use FFQ data to examine dietary patterns, derived from PCA, for children (of the women previously described) when aged 3, 4, 7 and 9 years and relate these to estimated nutrient intakes, using a well-characterised birth cohort study.

\section{Methods}

\section{Participants}

Participants were children participating in ALSPAC (www. alspac.bris.ac.uk), an ongoing longitudinal cohort study designed to investigate the health and development of children. The study has been described in more detail elsewhere ${ }^{(15)}$. Briefly, pregnant women were eligible if they had an expected delivery date between April 1991 and December 1992 and were resident in the former Avon Health Authority in South West England. This established a cohort of 14541 women resulting in 13988 children (alive at 12 months), with 548 new participants recruited at age 7 years (total children 14536). The primary source of data collection was via parental self-completion questionnaires. At recruitment the ALSPAC cohort was compared with the 1991 National Census data for mothers with infants aged 1 year resident in the area; they were similar except for a slight shortfall in those living in rented accommodation, single-parent families and ethnic minorities.

Ethical approval for the study was obtained from the ALSPAC Law and Ethics Committee and the Local Research Ethic Committees.

\section{Dietary assessment}

Dietary assessments were made using parent-completed FFQ throughout childhood when the children were aged $3 \cdot 2,4 \cdot 5,6 \cdot 8$ and $8 \cdot 6$ years, but for ease these time points will be referred to as 3, 4, 7 and 9 years, respectively. Each questionnaire contained a series of questions enquiring about the frequency of consumption of a wide range of food and drinks. These FFQ were modified from an original FFQ used to assess maternal diet at 32 weeks of pregnancy and shown to provide mean nutrient intakes $^{(16)}$ that were similar to those obtained in the UK National Diet and Nutritional Survey for women at that time $^{(17)}$. Over time the original FFQ was modified to include additional foods and drinks (with slight variations at each age) in the light of food record data collected from a sub-sample of ALSPAC children. Modifications included separate categories from age 4 years for vegetarian pies, coated white fish, coated chicken/turkey and tuna. These foods had previously been incorporated in other groups. School dinners (7 and 9 years only) were asked about separately and included in the nutrient analysis, but not the PCA.

The majority of the questions asked how many times the child consumed each food item 'nowadays' and the parent was asked to tick one of the following options: (i) 'never or rarely'; (ii) 'once in 2 weeks'; (iii) 'one to three times per week'; (iv) 'four to seven times per week'; or (v) 'more than once a day'. In addition, more detailed questions were asked about daily intakes of basic foods such as bread (the type and number of slices per day), tea and coffee (the number of cups). The type of milk (full-fat, semi-skimmed, others), bread (white, wholemeal, others) and spread (butter, margarine, others) usually consumed was also recorded. Standard portion sizes, modified according to the age of the child, were assumed throughout the questionnaires. Food records were used to give information on likely portion sizes and to inform the selection of foods to include in each food group ${ }^{(18,19)}$. Portion sizes were also informed by an analysis of weighed dietary intakes for similar aged children from the National Diet and Nutrition Survey ${ }^{(20-22)}$.

The weekly frequencies of intake assumed for each of the options in the FFQ were as follows: $\mathrm{i}=0$, $\mathrm{ii}=0.5$, iii $=2$, iv $=5.5$ and $v=10$ times/week. All items were standardised by subtracting the mean and dividing by the standard deviation for each variable. Some items were combined before the PCA, as they contributed to the same food group, e.g. 'Fish' in the PCA includes white fish, oily fish, shellfish and tuna. The final number of combined foods/food groups included in the PCA was thirty-four, thirty-five, forty-one and forty-one at 3, 4, 7 and 9 years, respectively.

Daily nutrient intakes were estimated from the FFQ using the fifth edition of McCance $\&$ Widdowson's The Composition of Food ${ }^{(23)}$ and supplements ${ }^{(24-29)}$. Additional up-to-date nutrient information was obtained from the National Diet and Nutrition Survey database and manufacturers' information ${ }^{(16)}$.

\section{Statistical analysis}

PCA with varimax rotation ${ }^{(30,31)}$ was performed separately at each time point on the standardised food items and has been described in detail elsewhere ${ }^{(10,32,33)}$. Briefly, PCA reduces the data by forming linear combinations of the original observed variables; thereby grouping together correlated variables, which in turn identifies any underlying dimensions in the data. The coefficients defining these linear combinations are called 'factor loadings' and are the correlations of each food item with that component. The number of components that best represented the data 
at each time point was chosen primarily on the basis of the scree plot ${ }^{(34)}$ and the interpretability of the factor loadings was also considered.

A score for each child was created for each component identified at each time point. These were calculated by multiplying the factor loadings by the corresponding standardised value for each food and summing across the food types. Each score has a mean of 0 (and a standard deviation of 1) and a higher score indicates that a child's diet is closer to that dietary pattern. Children were excluded from each PCA if they had more than ten items missing from the respective questionnaire. If ten or fewer items were missing, the assumption was made that the child did not consume those items and were given a value of 0 . Foods with loadings above 0.3 on a component were considered to have a strong association with that component and were deemed to be most informative in describing the dietary patterns. Labels were assigned to each component at each time point; these do not perfectly describe each underlying pattern, but were helpful in the reporting and the discussion of the results.

All component scores were approximately normally distributed. Spearman's correlation coefficients were calculated to measure the associations between the dietary pattern scores identified at each time point and the respective nutrient intakes. Additionally, partial correlation coefficients were calculated, adjusting for energy intake, and thus represented the associations between dietary patterns and relative nutrient intakes. Energy adjustment was conducted because energy is highly correlated with most other nutrients and related to body size ${ }^{(35)}$, thus it may obscure underlying relationships with nutrients. The nutrient density of an individual food is the ratio of its nutrient content to total energy content. Nutrient-dense foods provide substantial amounts of micronutrients with relative low energy content ${ }^{(36)}$; thus an overall diet that has a high micronutrient content after energy adjustment is more nutrient-dense and has a better nutrient profile. The proportion of variance explained by the dietary pattern scores was obtained for both absolute and energy-adjusted nutrient intakes by summing the squares of the correlations with each dietary pattern for each nutrient at each time point. All statistical analyses were performed using the statistical software package SPSS for Windows, version $12 \cdot 0$.

\section{Results}

\section{Response rates}

A total of $10133,9722,9515$ and 8331 questionnaires were returned at age 3, 4, 7 and 9 years, respectively, with $10023,9550,8286$ and 8010 having sufficient dietary data available at the respective ages for inclusion in the PCA. Three principal components - 'processed' (highfat/sugar processed foods, e.g. sausages, burgers, coated poultry, crisps, biscuits, sweets, chocolate and ice cream); 'health conscious' (vegetarian-style foods, salad, rice, pasta, fruit and fish); and 'traditional' (meat, potato and vegetables) - were consistently identified to best describe the dietary patterns of the children at the four time points throughout childhood $^{(10,32,33)}$. Note that at 9 years of age the 'health conscious' pattern was slightly different; meat and meat products had high negative loadings on this pattern and the name 'health conscious/vegetarian' was applied to reflect this change ${ }^{(33)}$.

\section{Correlations with energy}

The 'processed' pattern scores showed the highest correlations with energy intake at all ages $(r=0 \cdot 481-0 \cdot 616$; Table 1) with the 'health conscious' patterns showing the lowest correlations $(r=0 \cdot 034-0 \cdot 306$; Table 2$)$ and the 'traditional' pattern intermediate $(r=0 \cdot 219-0 \cdot 490$; Table 3).

\section{Associations between the 'processed' pattern and nutrient intakes}

Table 1 presents the correlations between the 'processed' dietary pattern scores and estimated nutrient intakes, both absolute and energy-adjusted, across the age groups. There were reasonably consistent correlations across the ages between this pattern and nutrient intakes. Strong positive correlations were observed between this dietary pattern and intakes of MUFA $(r=0.588-0 \cdot 708)$, SFA $(r=0 \cdot 468-0 \cdot 574)$ and sugar $(r=0 \cdot 475-0 \cdot 693)$ across all four time points that were attenuated but not removed by energy adjustment. At all ages, the majority of correlations with protein, fibre and micronutrients were reversed after energy adjustment. Thus higher scores on the 'processed' pattern were associated with poorer protein, fibre and micronutrient profile. The highest adjusted correlations were indeed negative and seen for $\mathrm{Mg}$, thiamin, niacin, $\mathrm{Zn}$ and fibre intake and the 'processed' dietary pattern score at each age ( $r$ ranging from -0.539 to -0.399 ).

\section{Associations between the 'bealth conscious' pattern and nutrient intakes}

The micronutrients rather than the macronutrients (except protein and fibre) were more strongly correlated with the 'health conscious' pattern, with the strength of the correlations lowest in the 9-year-olds (Table 2). Energy adjustment did not greatly attenuate the correlations between this pattern and protein, fibre or most micronutrients (e.g. the correlation with fibre at 3 years attenuated to 0.419 from 0.485 and at 7 years it attenuated to 0.475 from 0.537 ). However, correlations with some macronutrients were reversed; notably total fat, MUFA and SFA. In general higher scores on the health conscious' pattern were associated with a better protein, fibre and micronutrient profile. The highest adjusted correlations between the 'health conscious' pattern and various nutrients were seen at 3 years of age, but these were much lower by 9 years of age. For example, Mg reduced 
Table 1 Correlation coefficients between 'processed' dietary pattern scores across the ages and corresponding weekly absolute nutrient intakes and partial correlation coefficients between dietary pattern scores and weekly nutrient intakes adjusting for energy intake: children aged 3-9 years ( $n$ 8010 to 10 023), Avon Longitudinal Study of Parents and Children (ALSPAC), Bristol, UK

\begin{tabular}{|c|c|c|c|c|c|c|c|c|}
\hline \multirow[b]{2}{*}{ Nutrient } & \multicolumn{2}{|c|}{3 years } & \multicolumn{2}{|c|}{4 years } & \multicolumn{2}{|c|}{7 years } & \multicolumn{2}{|c|}{9 years } \\
\hline & Absolute & Adjusted & Absolute & Adjusted & Absolute & Adjusted & Absolute & Adjusted \\
\hline Energy & 0.481 & - & 0.693 & - & 0.619 & - & 0.619 & - \\
\hline Total fat & 0.529 & 0.259 & 0.662 & 0.065 & 0.604 & 0.085 & 0.600 & -0.060 \\
\hline Protein & 0.241 & -0.352 & 0.465 & -0.276 & 0.410 & -0.318 & 0.413 & 0.324 \\
\hline MUFA & 0.588 & 0.410 & 0.708 & 0.256 & 0.653 & 0.269 & 0.652 & 0.266 \\
\hline PUFA & 0.273 & -0.045 & 0.408 & 0.112 & 0.388 & $0 \cdot 118$ & 0.427 & 0.084 \\
\hline SFA & 0.468 & 0.138 & 0.574 & 0.017 & 0.555 & 0.084 & 0.543 & 0.050 \\
\hline Carbohydrate & 0.449 & -0.044 & 0.678 & 0.082 & 0.609 & 0.071 & 0.615 & 0.096 \\
\hline Sugar & 0.475 & $0 \cdot 150$ & 0.693 & 0.331 & 0.637 & 0.293 & 0.631 & $0 \cdot 278$ \\
\hline Fibre & 0.036 & -0.429 & 0.157 & -0.465 & 0.155 & -0.495 & $0 \cdot 191$ & -0.471 \\
\hline$n-3$ Fatty acids & -0.022 & -0.114 & 0.017 & -0.147 & -0.033 & -0.173 & 0.364 & -0.163 \\
\hline $\mathrm{K}$ & $0 \cdot 344$ & -0.148 & 0.519 & $-0 \cdot 125$ & 0.432 & -0.263 & $0 \cdot 610$ & -0.260 \\
\hline $\mathrm{Na}$ & 0.348 & -0.154 & 0.575 & -0.102 & 0.544 & -0.027 & 0.393 & 0.017 \\
\hline $\mathrm{Ca}$ & 0.288 & -0.168 & 0.486 & -0.144 & 0.466 & 0.000 & 0.418 & $-0 \cdot 197$ \\
\hline $\mathrm{Mg}$ & 0.103 & -0.539 & 0.314 & -0.483 & 0.307 & -0.503 & 0.646 & -0.492 \\
\hline $\mathrm{Fe}$ & $0 \cdot 190$ & -0.376 & 0.296 & -0.468 & 0.290 & -0.414 & 0.569 & -0.408 \\
\hline $\mathrm{Zn}$ & 0.173 & -0.464 & 0.323 & -0.470 & 0.338 & -0.399 & 0.674 & -0.507 \\
\hline Vitamin C & -0.139 & -0.351 & $0 \cdot 112$ & -0.194 & $0 \cdot 105$ & -0.193 & 0.497 & -0.177 \\
\hline Folate & $0 \cdot 105$ & -0.430 & 0.381 & -0.186 & 0.285 & -0.341 & 0.307 & -0.329 \\
\hline Carotene & -0.031 & -0.173 & 0.011 & -0.277 & -0.044 & -0.396 & -0.039 & -0.376 \\
\hline Retinol & 0.154 & -0.173 & 0.222 & -0.269 & 0.250 & $-0 \cdot 186$ & 0.229 & -0.263 \\
\hline Vitamin E & 0.212 & -0.056 & 0.304 & -0.142 & 0.320 & -0.070 & 0.354 & 0.058 \\
\hline Thiamin & 0.091 & -0.494 & 0.335 & -0.465 & 0.332 & -0.401 & 0.337 & -0.412 \\
\hline Niacin & 0.147 & -0.446 & 0.392 & -0.301 & 0.379 & -0.269 & 0.380 & -0.294 \\
\hline Riboflavin & 0.228 & -0.247 & 0.426 & 0.091 & 0.368 & -0.166 & 0.354 & -0.175 \\
\hline Vitamin $B_{6}$ & 0.241 & -0.270 & 0.519 & 0.009 & 0.473 & 0.057 & 0.422 & -0.200 \\
\hline
\end{tabular}

All $P<0.0001$.

Table 2 Correlation coefficients between 'health conscious" dietary pattern scores across the ages and corresponding weekly absolute nutrient intakes and partial correlation coefficients between dietary pattern scores and weekly nutrient intakes adjusting for energy intake: children aged 3-9 years ( $n 8010$ to 10 023), Avon Longitudinal Study of Parents and Children (ALSPAC), Bristol, UK

\begin{tabular}{|c|c|c|c|c|c|c|c|c|}
\hline \multirow[b]{2}{*}{ Nutrient } & \multicolumn{2}{|c|}{3 years } & \multicolumn{2}{|c|}{4 years } & \multicolumn{2}{|c|}{7 years } & \multicolumn{2}{|c|}{9 years* } \\
\hline & Absolute & Adjusted & Absolute & Adjusted & Absolute & Adjusted & Absolute & Adjusted \\
\hline Energy & $0 \cdot 278$ & - & 0.250 & - & 0.306 & - & 0.034 & - \\
\hline Total fat & 0.204 & -0.149 & $0 \cdot 183$ & -0.137 & 0.260 & -0.084 & 0.038 & -0.017 \\
\hline Protein & 0.383 & 0.289 & 0.246 & 0.070 & 0.245 & -0.041 & $-0 \cdot 164$ & -0.392 \\
\hline MUFA & $0 \cdot 165$ & -0.208 & $0 \cdot 117$ & -0.282 & $0 \cdot 191$ & -0.255 & -0.030 & $-0 \cdot 167$ \\
\hline PUFA & 0.289 & 0.152 & 0.251 & 0.115 & 0.274 & 0.078 & 0.024 & -0.003 \\
\hline SFA & $0 \cdot 141$ & -0.172 & $0 \cdot 124$ & -0.146 & 0.230 & -0.052 & 0.066 & 0.070 \\
\hline Carbohydrate & 0.262 & -0.013 & 0.262 & 0.080 & 0.317 & 0.088 & 0.083 & $0 \cdot 177$ \\
\hline Sugar & $0 \cdot 175$ & -0.111 & $0 \cdot 154$ & -0.072 & 0.254 & $0 \cdot 010$ & $0 \cdot 114$ & 0.149 \\
\hline Fibre & 0.485 & 0.419 & 0.559 & 0.529 & 0.537 & 0.475 & $0 \cdot 165$ & 0.203 \\
\hline$n-3$ Fatty acids & 0.340 & 0.312 & 0.284 & 0.252 & 0.270 & 0.234 & 0.040 & 0.035 \\
\hline $\mathrm{K}$ & 0.383 & 0.294 & 0.332 & $0 \cdot 210$ & $0 \cdot 319$ & $0 \cdot 114$ & -0.035 & -0.133 \\
\hline $\mathrm{Na}$ & 0.375 & 0.280 & 0.228 & 0.017 & 0.245 & -0.070 & -0.030 & -0.143 \\
\hline $\mathrm{Ca}$ & 0.304 & $0 \cdot 144$ & 0.287 & $0 \cdot 150$ & 0.339 & 0.173 & 0.147 & 0.193 \\
\hline $\mathrm{Mg}$ & 0.560 & 0.484 & 0.547 & 0.574 & 0.520 & 0.511 & $0 \cdot 149$ & 0.230 \\
\hline $\mathrm{Fe}$ & 0.426 & 0.356 & 0.467 & 0.436 & 0.468 & 0.388 & $0 \cdot 145$ & $0 \cdot 195$ \\
\hline $\mathrm{Zn}$ & 0.441 & 0.393 & 0.360 & 0.272 & $0 \cdot 342$ & $0 \cdot 165$ & -0.073 & $-0 \cdot 186$ \\
\hline Vitamin C & 0.508 & 0.464 & 0.438 & 0.387 & 0.406 & 0.326 & $0 \cdot 154$ & $0 \cdot 154$ \\
\hline Folate & 0.477 & 0.420 & 0.349 & 0.257 & 0.416 & 0.295 & 0.078 & 0.082 \\
\hline Carotene & 0.322 & 0.274 & 0.222 & $0 \cdot 160$ & $0 \cdot 291$ & $0 \cdot 195$ & -0.011 & -0.026 \\
\hline Retinol & 0.276 & $0 \cdot 147$ & 0.145 & 0.008 & 0.250 & 0.090 & 0.045 & 0.032 \\
\hline Vitamin E & 0.212 & 0.080 & $0 \cdot 184$ & 0.054 & 0.226 & 0.059 & 0.037 & 0.020 \\
\hline Thiamin & 0.414 & 0.326 & 0.345 & 0.247 & 0.343 & $0 \cdot 168$ & -0.004 & -0.059 \\
\hline Niacin & 0.368 & 0.253 & 0.259 & $0 \cdot 107$ & 0.223 & -0.047 & -0.196 & -0.396 \\
\hline Riboflavin & 0.268 & 0.091 & 0.247 & 0.098 & 0.300 & 0.115 & 0.065 & 0.058 \\
\hline Vitamin $\mathrm{B}_{6}$ & $0 \cdot 327$ & $0 \cdot 182$ & $0 \cdot 169$ & -0.031 & $0 \cdot 175$ & $-0 \cdot 127$ & $0 \cdot 151$ & -0.317 \\
\hline
\end{tabular}

All $P<0.0001$.

*'Health conscious/vegetarian' at the age of 9 years. 
Table 3 Correlation coefficients between 'traditional' dietary pattern scores across the ages and corresponding weekly absolute nutrient intakes and partial correlation coefficients between dietary pattern scores and weekly nutrient intakes adjusting for energy intake: children aged 3-9 years ( $n 8010$ to 10 023), Avon Longitudinal Study of Parents and Children (ALSPAC), Bristol, UK

\begin{tabular}{|c|c|c|c|c|c|c|c|c|}
\hline \multirow[b]{2}{*}{ Nutrient } & \multicolumn{2}{|c|}{3 years } & \multicolumn{2}{|c|}{4 years } & \multicolumn{2}{|c|}{7 years } & \multicolumn{2}{|c|}{9 years } \\
\hline & Absolute & Adjusted & Absolute & Adjusted & Absolute & Adjusted & Absolute & Adjusted \\
\hline Energy & $0 \cdot 219$ & - & 0.292 & - & $0 \cdot 340$ & - & 0.460 & - \\
\hline Total fat & $0 \cdot 182$ & -0.059 & 0.220 & -0.145 & 0.263 & -0.174 & 0.376 & -0.199 \\
\hline Protein & 0.483 & 0.579 & 0.575 & 0.624 & 0.599 & 0.646 & 0.642 & 0.554 \\
\hline MUFA & $0 \cdot 177$ & -0.057 & $0 \cdot 215$ & -0.131 & 0.267 & $-0 \cdot 131$ & 0.333 & -0.291 \\
\hline PUFA & 0.094 & -0.059 & $0 \cdot 153$ & -0.061 & 0.227 & -0.034 & 0.328 & -0.036 \\
\hline SFA & $0 \cdot 165$ & -0.034 & $0 \cdot 180$ & -0.107 & $0 \cdot 210$ & -0.147 & 0.305 & -0.172 \\
\hline Carbohydrate & $0 \cdot 145$ & -0.231 & 0.228 & $-0 \cdot 168$ & 0.284 & $-0 \cdot 152$ & 0.420 & -0.082 \\
\hline Sugar & $0 \cdot 165$ & -0.036 & $0 \cdot 174$ & -0.087 & 0.229 & -0.086 & 0.345 & -0.058 \\
\hline Fibre & 0.249 & 0.142 & 0.377 & 0.263 & 0.426 & 0.279 & 0.670 & 0.551 \\
\hline$n-3$ Fatty acids & $0 \cdot 175$ & $0 \cdot 145$ & 0.259 & 0.226 & 0.289 & 0.251 & 0.364 & 0.324 \\
\hline $\mathrm{K}$ & 0.396 & $0 \cdot 413$ & 0.523 & 0.519 & 0.516 & 0.472 & $0 \cdot 610$ & 0.482 \\
\hline $\mathrm{Na}$ & $0 \cdot 165$ & -0.050 & 0.226 & -0.067 & 0.296 & -0.019 & 0.393 & -0.064 \\
\hline $\mathrm{Ca}$ & 0.212 & 0.066 & 0.264 & 0.056 & 0.239 & -0.027 & 0.418 & $0 \cdot 107$ \\
\hline $\mathrm{Mg}$ & 0.226 & 0.085 & 0.360 & 0.222 & 0.418 & 0.260 & 0.646 & 0.547 \\
\hline $\mathrm{Fe}$ & 0.227 & 0.088 & 0.324 & $0 \cdot 167$ & 0.359 & $0 \cdot 157$ & 0.569 & 0.377 \\
\hline $\mathrm{Zn}$ & 0.403 & 0.406 & 0.554 & 0.542 & 0.552 & 0.515 & 0.677 & 0.598 \\
\hline Vitamin C & 0.244 & $0 \cdot 189$ & 0.328 & 0.252 & 0.358 & 0.260 & 0.497 & 0.379 \\
\hline Folate & 0.375 & 0.325 & 0.486 & 0.411 & 0.477 & 0.357 & 0.626 & 0.480 \\
\hline Carotene & 0.541 & 0.515 & 0.646 & 0.613 & 0.582 & 0.519 & 0.668 & 0.600 \\
\hline Retinol & 0.231 & 0.131 & 0.279 & 0.147 & 0.257 & 0.072 & 0.281 & 0.060 \\
\hline Vitamin E & 0.052 & -0.077 & $0 \cdot 102$ & -0.078 & $0 \cdot 151$ & 0.064 & 0.259 & -0.044 \\
\hline Thiamin & 0.328 & 0.256 & 0.449 & $0 \cdot 371$ & 0.475 & 0.366 & 0.593 & 0.427 \\
\hline Niacin & 0.485 & 0.527 & 0.540 & 0.515 & 0.593 & 0.575 & 0.628 & 0.495 \\
\hline Riboflavin & 0.289 & $0 \cdot 194$ & 0.337 & $0 \cdot 191$ & $0 \cdot 340$ & $0 \cdot 141$ & 0.445 & $0 \cdot 182$ \\
\hline Vitamin $\mathrm{B}_{6}$ & 0.471 & 0.503 & 0.532 & 0.497 & 0.536 & 0.541 & 0.572 & 0383 \\
\hline
\end{tabular}

All $P<0.0001$.

from $0 \cdot 484$ to $0 \cdot 230$; fibre from $0 \cdot 419$ to $0 \cdot 203$; vitamin $C$ from $0 \cdot 464$ to $0 \cdot 154$; and folate from $0 \cdot 420$ to $0 \cdot 082$.

\section{Associations between the 'traditional' pattern and nutrient intakes}

Table 3 shows the correlations between the 'traditional' dietary pattern scores and nutrient intakes across the ages. The 'traditional' pattern showed fairly consistent correlations with most nutrients, with a tendency for coefficients to increase with age (e.g. for carotene from 0.515 to $0 \cdot 600$ and for folate from 0.325 to $0 \cdot 480$ ). More than half of the nutrients examined at age 9 years were highly correlated with this pattern including protein, fibre, $\mathrm{K}, \mathrm{Mg}, \mathrm{Fe}, \mathrm{Zn}$, folate, carotene, thiamin, niacin and vitamin $\mathrm{B}_{6}$. These remained robust to energy adjustment. Correlations with some macronutrients were reversed after energy adjustment, such as with carbohydrate, sugar and all types of fat. The highest adjusted correlations were consistently seen for protein $(r=0.554-0.646)$, carotene $(r=0.515-0.613)$, $\mathrm{K}(r=0.413-0.519)$ and $\mathrm{Zn}(r=0.409-0.598)$. Higher scores on the 'traditional' pattern were consistently associated with greater protein, fibre and micronutrient density in the diet.

\section{Proportion of variance}

Table 4 indicates the proportion of the variance of each nutrient explained by the combined dietary patterns at each age. A reasonable proportion of the absolute intake of all macronutrients including fibre was explained by the dietary patterns at all ages. However, only fibre and protein were well explained after energy adjustment (37-54\%). For some micronutrients the dietary patterns explained a reasonable proportion of the variance at all ages, whether energy-adjusted or not; these were $\mathrm{K}, \mathrm{Mg}$, $\mathrm{Fe}, \mathrm{Zn}$, folate, thiamin and vitamin $\mathrm{B}_{6}$ (ranging from $25 \%$ to $86 \%$ ). The other micronutrients were not so well explained particularly after energy adjustment.

\section{Discussion}

The present study examining dietary data obtained from children at 3, 4, 7 and 9 years of age identified three consistent dietary patterns: 'processed', 'health conscious' and 'traditional'. These patterns taken together explained a high proportion of the variance in absolute intakes of energy, fibre and twenty-one nutrients at each age. However after energy adjustment only protein, fibre, $\mathrm{K}$, $\mathrm{Mg}, \mathrm{Fe}, \mathrm{Zn}$, folate, thiamin and vitamin $\mathrm{B}_{6}$ were well explained by the patterns (ranging from 25 to $86 \%$ ). Both the 'health conscious' and 'traditional' dietary patterns were positively associated with a better protein, fibre and micronutrient profile. On the other hand, high scores on the 'processed' pattern were associated with a worse protein, fibre and micronutrient profile at all ages. To our knowledge, the present study is the first one to explore 
Table 4 Proportion of variance (\%) explained for each nutrient by age at which dietary patterns were assessed: children aged 3-9 years ( $n 8010$ to 10 023), Avon Longitudinal Study of Parents and Children (ALSPAC), Bristol, UK

\begin{tabular}{|c|c|c|c|c|c|c|c|c|}
\hline \multirow[b]{2}{*}{ Nutrient } & \multicolumn{2}{|c|}{3 years } & \multicolumn{2}{|c|}{4 years } & \multicolumn{2}{|c|}{7 years } & \multicolumn{2}{|c|}{9 years } \\
\hline & Absolute & Adjusted & Absolute & Adjusted & Absolute & Adjusted & Absolute & Adjusted \\
\hline Energy & $35 \cdot 6$ & - & $63 \cdot 4$ & - & $59 \cdot 2$ & - & $59 \cdot 5$ & - \\
\hline Total fat & $35 \cdot 4$ & $9 \cdot 2$ & $51 \cdot 8$ & $4 \cdot 7$ & $50 \cdot 1$ & $4 \cdot 7$ & $50 \cdot 2$ & $7 \cdot 3$ \\
\hline Protein & $43 \cdot 8$ & $54 \cdot 2$ & $60 \cdot 7$ & $47 \cdot 6$ & $58 \cdot 6$ & $52 \cdot 6$ & $25 \cdot \overline{4}$ & $56 \cdot 5$ \\
\hline MUFA & $40 \cdot 4$ & $21 \cdot 4$ & $56 \cdot 1$ & $16 \cdot 2$ & $53 \cdot 4$ & $15 \cdot 4$ & $47 \cdot 5$ & $18 \cdot 3$ \\
\hline PUFA & $16 \cdot 6$ & $2 \cdot 8$ & $25 \cdot 2$ & $2 \cdot 9$ & $27 \cdot 7$ & $2 \cdot 5$ & $29 \cdot 3$ & $16 \cdot 5$ \\
\hline SFA & $26 \cdot 6$ & 4.9 & $37 \cdot 3$ & $3 \cdot 3$ & $40 \cdot 5$ & $3 \cdot 7$ & $39 \cdot 2$ & $3 \cdot 6$ \\
\hline Carbohydrate & $29 \cdot 0$ & $5 \cdot 5$ & $57 \cdot 6$ & $4 \cdot 1$ & $55 \cdot 2$ & $3 \cdot 5$ & $56 \cdot 1$ & $4 \cdot 7$ \\
\hline Sugar & $28 \cdot 3$ & $3 \cdot 6$ & $53 \cdot 4$ & $12 \cdot 2$ & $52 \cdot 2$ & $9 \cdot 3$ & $53 \cdot 0$ & $10 \cdot 2$ \\
\hline Fibre & $29 \cdot 8$ & $37 \cdot 5$ & $48 \cdot 2$ & $56 \cdot 5$ & $49 \cdot 3$ & $54 \cdot 6$ & $51 \cdot 2$ & $56 \cdot 6$ \\
\hline$n-3$ Fatty acids & $10 \cdot 2$ & $13 \cdot 1$ & $14 \cdot 8$ & $13 \cdot 6$ & $9 \cdot 0$ & $14 \cdot 7$ & $26 \cdot 6$ & $13 \cdot 2$ \\
\hline $\mathrm{K}$ & $42 \cdot 1$ & $27 \cdot 6$ & $64 \cdot 9$ & $32 \cdot 5$ & $55 \cdot 4$ & $51 \cdot 5$ & $67 \cdot 4$ & $31 \cdot 7$ \\
\hline $\mathrm{Na}$ & $28 \cdot 8$ & $10 \cdot 4$ & $43 \cdot 9$ & $1 \cdot 5$ & $44 \cdot 3$ & 0.5 & $24 \cdot 8$ & $2 \cdot 7$ \\
\hline $\mathrm{Ca}$ & $22 \cdot 0$ & $5 \cdot 3$ & $38 \cdot 8$ & $4 \cdot 6$ & 38.9 & $3 \cdot 6$ & $37 \cdot 1$ & $8 \cdot 7$ \\
\hline $\mathrm{Mg}$ & $37 \cdot 5$ & $53 \cdot 2$ & $52 \cdot 5$ & $61 \cdot 2$ & 53.9 & $56 \cdot 1$ & $85 \cdot 6$ & $59 \cdot 7$ \\
\hline $\mathrm{Fe}$ & $26 \cdot 9$ & $27 \cdot \overline{5}$ & $41 \cdot 0$ & $43 \cdot 7$ & $43 \cdot 2$ & $34 \cdot 6$ & $66 \cdot 8$ & $34 \cdot 6$ \\
\hline $\mathrm{Zn}$ & $38 \cdot 6$ & $53 \cdot 4$ & $54 \cdot 3$ & $58 \cdot 0$ & $53 \cdot 5$ & $45 \cdot 1$ & $76 \cdot 6$ & $64 \cdot 5$ \\
\hline Vitamin C & 3.9 & $37 \cdot 4$ & $31 \cdot 1$ & $25 \cdot 0$ & $30 \cdot 4$ & $21 \cdot 1$ & $51 \cdot 7$ & $19 \cdot 6$ \\
\hline Folate & $37 \cdot 9$ & $46 \cdot 6$ & $50 \cdot 3$ & $26 \cdot 5$ & $48 \cdot 1$ & $33 \cdot 6$ & $49 \cdot 2$ & $34 \cdot 5$ \\
\hline Carotene & $33 \cdot 4$ & $37 \cdot 0$ & $46 \cdot 6$ & $20 \cdot 2$ & $33 \cdot 5$ & $46 \cdot 4$ & $34 \cdot 6$ & $50 \cdot 2$ \\
\hline Retinol & $15 \cdot 3$ & $6 \cdot 6$ & $14 \cdot 4$ & $9 \cdot 4$ & $19 \cdot 1$ & $4 \cdot 7$ & $13 \cdot 3$ & 0.4 \\
\hline Vitamin E & $9 \cdot 2$ & 1.5 & $13 \cdot 6$ & $2 \cdot 5$ & $17 \cdot 6$ & $1 \cdot 2$ & $19 \cdot 3$ & 0.5 \\
\hline Thiamin & $28 \cdot \overline{7}$ & $41 \cdot 5$ & $43 \cdot 2$ & $41 \cdot 4$ & $45 \cdot 3$ & $32 \cdot 2$ & $45 \cdot 7$ & $35 \cdot 5$ \\
\hline Niacin & $39 \cdot 2$ & $54 \cdot 6$ & $51 \cdot 2$ & $36 \cdot 7$ & $54 \cdot 5$ & $40 \cdot 6$ & $14 \cdot 6$ & $48 \cdot 6$ \\
\hline Riboflavin & $20 \cdot 7$ & $10 \cdot 6$ & $36 \cdot 2$ & $7 \cdot 6$ & $34 \cdot 1$ & $6 \cdot 6$ & $32 \cdot 7$ & $6 \cdot 7$ \\
\hline Vitamin $\mathrm{B}_{6}$ & 38.6 & 35.5 & $57 \cdot 6$ & $24 \cdot 6$ & $54 \cdot 1$ & $24 \cdot 6$ & $52 \cdot 8$ & $28 \cdot 7$ \\
\hline
\end{tabular}

the relationship between dietary patterns using PCA and nutrients at multiple time points throughout childhood.

The fact that the dietary patterns were able to explain a large proportion of the variance of many nutrients adds credibility to the use of these types of pattern to summarise diet. In the present study nine of the twenty-four nutrients were particularly well explained, with a quarter to a half of their variance explained at each age, whether energy-adjusted or not. These were protein, fibre, $\mathrm{K}, \mathrm{Mg}$, $\mathrm{Fe}$, folate, $\mathrm{Zn}$, thiamin and vitamin $\mathrm{B}_{6}$. These are all vital nutrients and $\mathrm{Zn}, \mathrm{Fe}$ and fibre have been shown to be marginal in the diets of children. $\mathrm{Zn}$ is required for growth, development and cognitive function ${ }^{(37)} ; \mathrm{Fe}$ is also essential for cognitive functioning and low levels are an indication of poor nutrition ${ }^{(38)}$; while fibre is needed for digestion, helps control appetite and is important in determining obesity risk ${ }^{(39)}$.

Clear positive correlations, which were fairly robust to energy adjustment, were evident between the scores on the 'processed' pattern and intakes of MUFA, SFA and sugar at all ages. Furthermore, intakes of almost all of the micronutrients as well as fibre and protein were negatively correlated with this pattern after energy adjustment. This result is not surprising given that high scores on the 'processed' pattern are associated with high consumption of high-fat/sugar, nutrient-poor processed foods such as chips, pizza, meat pies, sausages and burgers, as well as crisps, biscuits, sweets, chocolate and ice creams. This pattern, characterised by an elevated intake of both fat and sugar, is likely to contribute to the increasing prevalence of childhood obesity ${ }^{(40)}$ and may play an important role in high blood pressure; a risk factor for $\mathrm{CVD}^{(41)}$. The fact that the correlations of this pattern with energy were very strong and those with micronutrients were reversed after energy adjustment suggests that it is a marker for an energy-dense, nutrient-poor diet.

The 'health conscious' pattern was the least correlated of the three patterns with energy and showed positive relationships with fibre and many micronutrients, such as vitamin $\mathrm{C}, \mathrm{Mg}$, Fe and $\mathrm{Zn}$, that were robust to energy adjustment. This result was again not unexpected given that higher pattern scores are related to higher intakes of nutrient-dense foods such as fruit, vegetables, breakfast cereals, fish and pulses. However, these relationships were not as evident in the 9-year data. This could be because the 'health conscious' pattern had a slightly different character at this age; in particular, there was a negative association with meat which had not been present at previous ages ${ }^{(33)}$. It is likely that the associations with $\mathrm{Fe}$ and $\mathrm{Zn}$ would have been weakened by this. On the whole, however, this pattern is a potential marker of increased diet quality with high scores associated with a better micronutrient profile in the diet.

The 'traditional' pattern showed fairly consistent correlations that strengthened a little as the children became older. Higher scores on this pattern are primarily associated with higher meat and vegetable intakes, so the robust positive correlations with protein, $\mathrm{K}, \mathrm{Zn}$, carotene and niacin are sensible. The reversal of associations with energy-dense macronutrients such as fat, carbohydrate 
and sugar after energy adjustment and the continued positive relationship with almost all macronutrients suggest that this pattern is also a marker for a diet with a better nutrient profile, and the consumption of foods associated with this pattern, particularly vegetables, needs to be encouraged.

Dietary patterns have, in recent times, become accepted as an alternative or complementary approach to the more traditional methods of assessing the associations between diet and disease ${ }^{(12)}$. Previous work exploring these associations in relation to estimated nutrient intakes, although still limited, has primarily been in adults ${ }^{(42-45)}$. To our knowledge, only one UK study has examined the associations between dietary patterns and nutrient intakes in children. Patel et al. (2011) obtained three dietary patterns in a sample of 709 children aged 11 years. They showed that the 'fruit' pattern was inversely correlated with fat intakes, while the opposite trend was observed for the 'processed' pattern. Both vitamins $\mathrm{C}$ and $\mathrm{E}$ had positive associations with the 'fruit' and 'traditional' patterns, but an inverse relationship was seen with the 'processed' pattern ${ }^{(46)}$. These finding are very similar to those in the current study.

Another study among schoolchildren (aged 6-18 years) in Bahrain used energy and macronutrient intakes to determine dietary patterns (as opposed to food groups); it found a dietary pattern that was associated with high sugar intake, a high percentage of energy from saturated fat and low fibre intake. Children who scored highly on this pattern were more likely to consume sweets, snacks and soft drinks, but less likely to consume milk, fruit and vegetables. The authors suggested that this pattern is likely to contribute to the risk of obesity and other health problems in later life ${ }^{(47)}$. In recent times in Bahrain, there has been a considerable shift away from traditional foods and towards diets containing high amounts of fat, sugar and red meat ${ }^{(48)}$.

The results observed in the current study were consistent with results obtained in pregnant women (the mothers of the children reported here), which found a 'processed' pattern that was positively associated with fats and sugar, as well as 'health conscious' and 'traditional' patterns that both showed positive linear relationships with most nutrients ${ }^{(14)}$. Similar results have also been presented in other adult studies $^{(42-45)}$. Arrkola et al. (2008) reported dietary patterns in relation to dietary intakes in 3730 pregnant women and identified seven dietary patterns, with energy intake found to be positively correlated with the 'healthy', 'fast food', 'traditional bread', 'traditional meat' and 'coffee' patterns. As expected, the 'fast food' pattern was associated primarily with carbohydrate, fat and saturated fat, while the 'healthy' pattern was positively correlated with vitamin C, folate, Fe, $\mathrm{Mg}$ and protein among others. Fibre intake was positively correlated with the 'traditional bread' pattern together with protein, carbohydrate, fat, folate and $\mathrm{Fe}^{(42)}$. Using data from the European Prospective Investigation into Cancer and
Nutrition (EPIC), Schulze et al. (2001) found in both men and women that most of the seven dietary patterns identified were positively associated with energy intake; a strong correlation between the 'sweets' pattern and carbohydrate intake, but not fat or protein intake, remained after energy adjustment; the 'fruit and vegetables' pattern had a positive relationship with fibre, Fe and vitamin $\mathrm{C}$ in both sexes; and the 'bread and sausage' pattern in men was associated with salt intake ${ }^{(45)}$. Results from the current study and others investigating dietary patterns and nutrient intakes have the potential to inform new public health initiatives, as this method of examining dietary patterns as a whole can be more useful than focusing on single foods and/or nutrients ${ }^{(12)}$. It is evident from the results presented here that the 'processed' pattern is the least ideal in terms of nutrient intake and this persists through early to mid-childhood. By encouraging our children to reduce their intake of fats and sugars (thereby scoring lower on the 'processed' pattern) we have the potential to improve their nutrient profile and therefore their health. Conversely, by encouraging increased fruit and vegetable intake (thereby moving towards our 'health conscious' or 'traditional' pattern) nutrient intakes can be improved.

The strengths of the current study are its large sample size and its longitudinal design, which allowed dietary patterns and nutrient intakes to be examined in the same children throughout childhood. However, there are several limitations. First, it was conducted in one geographic area of England and so results may not be applicable throughout the UK. However, the cohort was reasonably representative of the UK population at recruitment. Second, dietary intakes were obtained using an unquantified FFQ which is a less accurate method of dietary assessment than using weighed dietary records ${ }^{(49)}$. Nevertheless, FFQ have been shown to provide a reasonable measure of diet in large cohorts ${ }^{(50)}$ and may in fact estimate intakes of less commonly eaten foods more accurately than dietary records. We chose to examine the data cross-sectionally at each time point as the dietary patterns changed slightly at each age, no doubt due to the children progressing through different life stages from pre-school through to starting school and moving on into middle childhood with different influences being felt. In order to perform longitudinal analysis we would have needed to impose the pattern scores from the diet at 3 years on to the later data, and we felt that this was inappropriate due to the changing circumstances of the children.

\section{Conclusions}

We have shown that dietary patterns throughout childhood have similar relationships with estimated nutrient intakes at each age. It is noteworthy that the 'traditional' and 'health conscious' patterns showed very similar relationships and the 'traditional' dietary pattern should 
not be dismissed as a 'nutritiously poor' pattern in studies assessing diet and health outcomes. The construction of dietary patterns can be a useful way of summarising diet which has a credible relationship with important nutrients and with diet quality. Their use in dietary investigation should be helpful in providing evidence of how diet interacts with later health outcomes ${ }^{(12)}$.

\section{Acknowledgements}

Source of funding: This specific research was funded by the World Cancer Research Fund (grant number 2009/23). The UK Medical Research Council, the Wellcome Trust and the University of Bristol provide core support for ALSPAC. Conflict of interest: The authors declare no conflict of interest. Authors' contribution: P.E. collected the data, K.N. conceived the study design and performed the analyses, V.C. wrote the first draft of the manuscript and all authors approved the final version. This publication is the work of all of the authors and K.N. serves as guarantor for the contents of this paper. Acknowledgements: The authors are extremely grateful to all families who took part in the study, the midwives for their help in recruiting them and the whole ALSPAC team, which includes interviewers, computer and laboratory technicians, clerical workers, research scientists, volunteers, managers, receptionists and nurses.

\section{References}

1. Dietz WH (1994) Critical periods in childhood for the development of obesity. Am J Clin Nutr 59, 955-959.

2. Dietz WH (2001) The obesity epidemic in young children. BMJ 322, 313-314.

3. Steyn NP, Mann J, Bennett PH et al. (2004) Diet, nutrition and the prevention of type 2 diabetes. Public Health Nutr 7, $147-165$.

4. World Cancer Research Fund \& American Institute for Cancer Research (1997) Food, Nutrition and the Prevention of Cancer: A Global Perspective. Washington, DC: American Institute for Cancer Research.

5. World Health Organization (2003) Diet, Nutrition and the Prevention of Chronic Diseases. Joint WHO/FAO Expert Consultation. WHO Technical Report Series no. 916. Geneva: WHO.

6. Mikkila V, Rasanen L, Raitakari OT et al. (2005) Consistent dietary patterns identified from childhood to adulthood: the Cardiovascular Risk in Young Finns Study. Br J Nutr 93, 923-931.

7. Craig LCA, McNeill G, Macdiarmid JI et al. (2010) Dietary patterns of school-age children in Scotland: association with socio-economic indicators, physical activity and obesity. Br J Nutr 103, 319-334.

8. Hu FB (2002) Dietary pattern analysis: a new direction in nutritional epidemiology. Curr Opin Lipidol 13, 3-9.

9. Michels KB \& Schulze MB (2005) Can dietary patterns help us detect diet-disease associations? Nutr Res Rev 18, 241-248.

10. North K, Emmett P \& the ALSPAC Study Team (2000) Multivariate analysis of diet among three-year-old children and associations with socio-demographic characteristics. Eur J Clin Nutr 54, 73-80.
11. Jacques PF \& Tucker KL (2001) Are dietary patterns useful for understanding the role of diet in chronic disease? Am J Clin Nutr 73, 1-2.

12. Cutler GJ, Flood A, Hannan P et al. (2009) Major patterns of dietary intake in adolescents and their stability over time. J Nutr 139, 323-328.

13. Mikkila V, Rasanen L, Raitakari OT et al. (2007) Major dietary patterns and cardiovascular risk factors from childhood to adulthood. The Cardiovascular Risk in Young Finns Study. Br J Nutr 98, 218-225.

14. Northstone K, Emmett PM \& Rogers I (2008) Dietary patterns in pregnancy and associations with nutrient intakes. Br J Nutr 99, 406-415.

15. Golding J, Pembrey M, Jones R et al. (2001) ALSPAC - The Avon Longitudinal Study of Parents and Children I. Study methodology. Paediatr Perinat Epidemiol 15, 74-87.

16. Rogers IS, Emmett P, Golding J et al. (1998) Diet during pregnancy in a population of pregnant women in South West England. Eur J Clin Nutr 52, 246-250.

17. Ministry of Agriculture, Fisheries and Food (1994) The Dietary and Nutritional Survey of British Adults: Further Analysis. London: HMSO.

18. Emmett PM, Rogers IS, Symes C et al. (2002) Food and nutrient intakes of a population sample of 3-year-old children in the South West of England in 1996. Public Health Nutr 5, 55-64.

19. Glynn L, Emmett P, Rogers I et al. (2005) Food and nutrient intakes of a population sample of 7-year-old children in the south-west of England in 1999/2000 - what difference does gender make? J Hum Nutr Diet 18, 7-19.

20. Gregory J \& Lowe S (2000) National Diet and Nutrition Survey: Young People Aged 4 to 18 Years. vol. 1: Report of the Diet and Nutrition Survey. London: The Stationery Office.

21. Gregory JR, Collins DL, Davies PSW et al. (1995) The National Diet and Nutrition Survey Children Aged 1 1/2 to 4 1/2 Years. vol. I: Report of the Diet and Nutrition Survey. London: HMSO.

22. Wrieden WL, Longbottom PJ, Adamson AJ et al. (2008) Estimation of typical food portion sizes for children of different ages in Great Britain. BrJ Nutr 99, 1344-1353.

23. Holland B, Welch AA, Unwin ID et al. (1991) McCance \& Widdowson's The Composition of Foods, 5th ed. Cambridge: The Royal Society of Chemistry.

24. Chan W, Brown J \& Buss DH (1994) Miscellaneous Foods. Fourth Supplement to 5th edition of McCance \& Widdowson's The Composition of Foods. Cambridge: The Royal Society of Chemistry.

25. Chan W, Brown J, Church SM et al. (1996) Meat Products and Dishes. Sixth Supplement to 5th edition of McCance \& Widdowson's The Composition of Foods. Cambridge: The Royal Society of Chemistry.

26. Holland B, Brown J \& Buss DH (1993) Fish and Fish Products. Third Supplement to 5th edition of McCance \& Widdowson's The Composition of Foods. Cambridge: The Royal Society of Chemistry.

27. Holland B, Welch AA \& Buss DH (1992) Vegetable Dishes. Second Supplement to 5th edition of McCance \& Widdowson's The Composition of Foods. Cambridge: The Royal Society of Chemistry.

28. Holland B, Unwin ID \& Buss DH (1992) Fruit and Nuts. First Supplement to 5th edition of McCance \& Widdowson's The Composition of Foods. Cambridge: The Royal Society of Chemistry.

29. Holland B, Unwin ID \& Buss DH (1991) Vegetables, Herbs and Spices. Fifth Supplement to McCance \& Widdowson's The Composition of Foods, th ed. Cambridge: The Royal Society of Chemistry.

30. Gorsuch RL (1974) Factor Analyses. Philaelphia, PA: W.B. Saunders. 
31. Kline P (1994) An Easy Guide to Factor Analysis. London: Routledge.

32. Northstone K, Emmett P \& the ALSPAC Study Team (2005) Multivariate analysis of diet in children at four and seven years of age and associations with socio-demographic characteristics. Eur J Clin Nutr 59, 751-760.

33. Northstone K \& Emmett P (2008) Are dietary patterns stable throughout early and mid-childhood? Br J Nutr 100, 1069-1076.

34. Cattell RB (1966) The scree test for the number of factors. Multivariate Behav Res 1, 245-276.

35. Willett WC, Howe GR \& Kushi LH (1997) Adjustment for total energy intake in epidemiologic studies. Am J Clin Nutr 65, 4 Suppl., S1220-S1228.

36. US Department of Agriculture \& US Department of Health and Human Services (2010) Dietary Guidelines for Americans 2010. Washington, DC: US Government Prininting Office.

37. Salgueiro MJ, Zubillaga MB, Lysionek AE et al. (2002) The role of zinc in the growth and development of children. Nutrition 18, 510-519.

38. De Benoist B, McLean E, Egli I et al. (2008) Worldwide Prevalence of Anaemia 1993-2005: WHO Global Database on Anaemia. Geneva: WHO.

39. Johnson L, Mander AP, Jones LR et al. (2008) Energy-dense, low-fiber, high-fat dietary pattern is associated with increased fatness in childhood. Am J Clin Nutr 87, 846-854.

40. Bray GA, Paeratakul S \& Popkin BM (2004) Dietary fat and obesity: a review of animal, clinical and epidemiological studies. Physiol Behav 83, 549-555.

41. He FJ \& MacGregor GA (2010) Reducing population salt intake worldwide: from evidence to implementation. Prog Cardiovasc Dis 52, 363-382.
42. Arkkola T, Uusitalo U, Kronberg-Kippilae C et al. (2008) Seven distinct dietary patterns identified among pregnant Finnish women - associations with nutrient intake and sociodemographic factors. Public Health Nutr 11, 176-182.

43. Crozier SR, Robinson SM, Borland SE et al. (2006) Dietary patterns in the Southampton Women's Survey. Eur J Clin Nutr 60, 1391-1399.

44. Randall E, Marshall J, Graham S et al. (1990) Patterns in food use and their associations with nutrient intakes. $A m J$ Clin Nutr 52, 739-745.

45. Schulze MB, Hoffmann K, Kroke A et al. (2001) Dietary patterns and their associations with food and nutrient intake in the European Prospective Investigation into Cancer and Nutrition (EPIC) - Potsdam study. Br J Nutr 85, 363-373.

46. Patel S, Murray CS, Simpson A et al. (2011) Dietary patterns of 11-year-old children and associations with nutrient intakes. J Hum Nutr Diet 24, 399-400.

47. Gharib N \& Rasheed P (2011) Energy and macronutrient intake and dietary pattern among school children in Bahrain: a cross-sectional study. Nutr J 10, 62.

48. Musaiger AO (2000) The state of nutrition in Bahrain. Nutr Health 14, 63-74.

49. Bingham SA, Gill C, Welch A et al. (1994) Comparison of dietary assessments methods in nutritional epidemiology: weighed records $v .24 \mathrm{~h}$ recalls, food-frequency questionnaires and estimated-diet records. Br J Nutr 72, 619-643.

50. Willett W \& Stampfer M (1998) Implications of total energy intake for epidemiologic analysis. In Nutritional Epidemiology, 2nd ed., pp. 273-301 [W Willett, editor]. New York: Oxford University Press. 